PROCEEDINGS OF THE

AMERICAN MATHEMATICAL SOCIETY

Volume 34, Number 2, August 1972

\title{
LOCAL TRIVIALITY OF FIBERINGS
}

\author{
SOON-KYU KIM ${ }^{1}$
}

\begin{abstract}
We prove that a Hurewicz fibering or a Serre fibering is locally trivial if the total space is a connected separable metric ANR $n$-gm over a principal ideal domain and the base space is a weakly locally contractible paracompact finite dimensional space, and all fibers are homeomorphic to a space which is a connected 3-manifold with exactly one end and whose one point compactification is a 3 -manifold and it has no false 3 -cells, in particular a euclidean 3-space.
\end{abstract}

1. Introduction. A map $p: E \rightarrow B$ is a Hurewicz fiber map if $p$ has the covering homotopy property for all topological spaces and it is a Serre fiber map if $p$ has the covering homotopy property for polyhedrons. In [10], Raymond conjectured that a Hurewicz fiber map is locally trivial if the total space $E$ is a manifold without boundary and the base space $B$ is a weakly locally contractible (wlc) paracompact space. In supporting the conjecture, Raymond proved that a Hurewicz fiber map is locally trivial if $E$ is a connected separable metric ANR (generalized) manifold (over a principal ideal domain) and $B$ is a wlc paracompact space and it has a fiber which contains a compact connected component of dimension $\leqq 2$.

The conjecture is false if $E$ is allowed to have nonempty boundary or if $E$ is not a manifold. The latter is due to the replacement theorem of Fadell, Langston and Tulley [8]. However, Kim extended the result of Raymond to the case where the manifold $E$ has nonempty boundary by imposing more conditions on the fibering, and to the case where the fibers are noncompact manifolds [6]. In particular, Kim proved that a Hurewicz fibering is locally trivial if all fibers are homeomorphic to either $R^{1}$ or $R^{2}$ (euclidean spaces) and conjectured that a Hurewicz fibering is locally trivial if all fibers are homeomorphic to $R^{3}$.

In this paper, we settle the above conjecture of Kim affirmatively. We actually prove a slightly more general theorem, as we have already done

Received by the editors July 15, 1971.

AMS 1969 subject classifications. Primary 5550, 5560.

Key words and phrases. Hurewicz fiber map, Serre fiber map, homotopically $n$-regular map, locally trivial map, $n$-gm, wlc, ends, one-point compactification.

${ }_{1}^{1}$ Partially supported by grant NSF GP-29117.

(c) American Mathematical Society 1972 
elsewhere in the case where the fibers are $R^{2}$. A similar thing can be said for a Serre fiber map. The method of proof is quite similar to the case where the fibers are $R^{2}$ [6]. We compactify the total space $E$ along each fiber and we use the results of Dyer and Hamstrom, namely the facts that a homotopically 2-regular map is a completely regular map when the dimension of fibers is low and a completely regular map is locally trivial when the dimension of fibers is low ([2], [4], and [5]).

In [7], Kim also extended the Dyer and Hamstrom result that a completely regular map is locally trivial to the case of higher dimensional fibers. However, we cannot extend our present result to the case where the fibers are $R^{n}(n>3)$ because we do not know how to go by the first result of Dyer and Hamstrom to the case where the dimension of the fiber is big.

2. Construction. Let $p: E \rightarrow B$ be a Hurewicz fiber map from a connected separable metric ANR $n$-gm $E$ over a principal ideal domain onto a wlc paracompact finite (covering) dimensional base space $B$. By a generalized $n$-manifold ( $n$-gm) we mean what Wilder and Raymond call a (locally orientable) cohomology $n$-manifold (see [10] and [14]). Suppose that all fibers are homeomorphic to a space $M$, where $M$ is a connected 3-manifold with exactly one end (see [11] for definition) and whose one point compactification is a 3 -manifold. We note that $B$ is necessarily 0 -connected and is a separable metric ANR [3], and $B$ is also locally compact because $E$ is locally compact and the map $p: E \rightarrow B$ is open [3].

Let $\bar{E}$ be the disjoint union of $E$ and the product space $B \times\{0\}$. We define $\bar{p}: \bar{E} \rightarrow B$ by $\bar{p}(e)=p(e)$ for each $e \in E$ and $\bar{p}(e)=b$ for each $e=(b, 0) \in$ $B \times\{0\}$. We give a topology $\overline{\mathscr{U}}$ on $\bar{E}$ in the following way: Let $\mathscr{U}$ be the collection of all open sets of $E$. Let $W$ be an open set of $B$ such that $p$ admits a cross section $f$ on $W$; furthermore there exists a closed subset $W_{0}$ of $p^{-1}(W)$ such that the closure of $W_{0}$ in $E$ is compact and $W_{0} \supset f(W)$ and $W_{0} \cap p^{-1}(b)$ is compact in $p^{-1}(b)$ for each $b \in W$. To see that such $W$ and $W_{0}$ exist, we use the local contractibility of $B$ and a fiber homotopy equivalence between $p^{-1}\left(W^{\prime}\right)$ and $W^{\prime} \times p^{-1}\left(b_{0}\right)$ where $b_{0} \in W^{\prime}$ and $W^{\prime}$ is an open subset of $B$ which is uniformly contractible in $B$ (see [6, p. 59] for details). There are infinitely many such pairs $\left(W, W_{0}\right), W \ni b$, for each point $b \in B$. The collection of all such pairs for all $b \in B$ will be denoted by $\mathscr{C}$. Let $\mathscr{V}^{\prime}$ be the collection of sets of the form $\bar{p}^{-1}(W)-W_{0}$ for each $\left(W, W_{0}\right) \in \mathscr{C}$, and let $\mathscr{V}$ be the collection of all subsets $V$ of $\bar{E}$ such that $V \cap E$ is open in $E$ and $V \cap(\bar{E}-E) \neq \varnothing$, and for each $x \in$ $V \cap(\bar{E}-E)$ there exists an element $V^{\prime} \in \mathscr{V}^{\prime}$ such that $x \in V^{\prime} \subset V$. Then any element $V^{\prime}$ of $\mathscr{V}^{\prime}$ is an element of $\mathscr{V}$ (see [6]). Now let $\overline{\mathscr{U}}$ denote the collection of all elements of $\mathscr{U}$ and $\mathscr{V}$. Then it is easy to see that $\overline{\mathscr{U}}$ is a topology on $\bar{E}$. Henceforth $\bar{E}$ denotes a topological space with the topology $\overline{\mathscr{U}}$. 
2.1. Lemma. (i) The map $\bar{p}: \bar{E} \rightarrow B$ is continuous and open,

(ii) the subspace $B \times\{0\}$ of $\bar{E}$ is homeomorphic to $B$,

(iii) the space $\bar{E}$ is a locally compact Hausdorff space,

(iv) the space $\bar{E}$ is a topologically complete metric space,

(v) the space $\bar{p}^{-1}(b)$, as a subspace of $\bar{E}$, is the one point compactification of $p^{-1}(b)$ for each $b \in B$, hence $\bar{p}^{-1}(b)$ is a compact 3-manifold with added point as its interior point,

(vi) the map $\bar{p}: \bar{E} \rightarrow B$ is a proper map.

Proof. Some of these properties are direct consequences of the definition of the space $\bar{E}$ and the map $\bar{p}: \bar{E} \rightarrow B$ and nontrivial facts were proved in [6, p. 60] (see also [12]).

We recall here the definition of the homotopic $n$-regularity of a map. A map $f$ from a metric space $X$ onto a metric space $Y$ is homotopically $n$-regular if it is open and proper and if for given $x \in X$ and $\varepsilon>0$ there exists $\delta>0$ such that each mapping of a $k$-sphere $S^{k}, k \leqq n$, into $S(x, \delta) \cap$ $f^{-1}(y), y \in Y$, is homotopic to a constant map in $S(x, \varepsilon) \cap f^{-1}(y)$, where $S(x, \varepsilon)$ is the $\varepsilon$-neighborhood of $x$.

\subsection{Lemma. The map $\bar{p}: \bar{E} \rightarrow B$ is homotopically 2-regular.}

Proof. By (2.5) of [10], $p: E \rightarrow B$ is a homotopically 2-regular map without assuming the properness. Therefore, it suffices to verify the conditions of the homotopic 2-regularity of $\bar{p}$ on the points in $\bar{E}-E$. But this follows by our definition of the neighborhood systems of points in $\bar{E}-E$ which are defined "fiber-wise" in a sense. More specifically, let a point $x \in(\bar{E}-E)$ and $\varepsilon>0$ be given. Since $S(x, \varepsilon)$ is open in $\bar{E}$, it contains an element $V \in \mathscr{V}$ which is an open neighborhood of $x$ such that $V=$ $\bar{p}^{-1}(W)-W_{0}$ for an element $\left(W, W_{0}\right) \in \mathscr{C}$, where $\bar{p}(x) \in W$. Since $\bar{p}^{-1}(\bar{p}(x)) \cap$ $V$ is open in $\bar{p}^{-1}(\bar{p}(x))$ and $x$ is an interior point of a 3-manifold by Lemma 2.1, there exists a closed 3-cell $D_{x}$ of a positive diameter around $x$ in $\bar{p}^{-1}(\bar{p}(x))$ such that $D_{x} \subset V$; i.e., $D_{x}=S(x, \alpha) \cap \bar{p}^{-1}(\bar{p}(x))$ where $\alpha$ is a number. Furthermore, $V$ is an open neighborhood of $y=\bar{p}^{-1}(c) \cap(\bar{E}-E)$ and $\bar{p}^{-1}(c) \cap V$ is open in $\bar{p}^{-1}(c)$ for each $c \in W$. Therefore, we can find a neighborhood $U$ of $x$ in $\bar{E}-E$ so that for each $y \in U$, there exists a closed 3-cell $D_{y}$ around $y$ in $\bar{p}^{-1}(\bar{p}(y))$ whose diameter is bigger than some number $\alpha>0$ and $D_{y} \subset V$. If $\alpha$ is once chosen we may as well assume that the distance from $x$ to the frontier of $U$ denoted by $d(x, \operatorname{Fr}(U))$ is less than $\alpha / 4$ by changing $U$ if necessary. Now since $\bar{p}(U)$ is again open in $B$, $d\left(x, \bar{p}^{-1}(\bar{p}(\operatorname{Fr}(U)))\right)=\beta$ is nonzero. Take $\delta$ to be the minimum number of $\beta$ and $\alpha / 4$. Then the open neighborhood $S(x, \delta)$ of $x$ is contained in the union of all $D_{y}, y \in U$, hence in $V$. For if $z \in S(x, \delta)$ then $d(x, z)<\delta \leqq \beta$. Therefore, first of all $z \in \bar{p}^{-1}(\bar{p}(U))$; i.e., $\bar{p}^{-1}(\bar{p}(z)) \cap(\bar{E}-E)=y \in U$. 
Furthermore, $d(y, z) \leqq d(y, x)+d(x, z)<\alpha / 4+\alpha / 4=\alpha / 2$, and hence $z \in$ $\operatorname{Int}\left(D_{y}\right)$.

Then if $S(x, \delta) \cap \bar{p}^{-1}(c)$ is nonempty, any map $g: S^{k} \rightarrow S(x, \delta) \cap \bar{p}^{-1}(c)$ is homotopic to a constant map in $S(x, \varepsilon)$ for all $k$ since $S(x, \delta) \cap \bar{p}^{-1}(c) \subset$ Int $\left(D_{y}\right) \subset V \subset S(x, \varepsilon)$ and $D_{y}$ is a 3-cell, where $y \in(\bar{E}-E)$ with $\bar{p}(y)=c$ in $B$.

We know that $\bar{p}$ is an open and proper map by Lemma 2.1. Therefore $\bar{p}$ is a homotopically 2-regular map (in fact, $n$-regular). tion.

We prove another lemma which is independent of the above construc-

2.3. LEMMA. Let $M$ be a 3-manifold whose one point compactification $M^{+}$is again a 3-manifold. If all homotopy 3-cells in $M$ are 3-cells then all homotopy 3-cells in $\mathrm{M}^{+}$are 3-cells too.

Proof. It suffices to consider a homotopy 3-cell $D$ in $M^{+}$such that $x \in \operatorname{Int}(D)$. Choose an $\operatorname{arc} A$ from $x$ to a point of the boundary of $D$, and a nice tabular neighborhood $N$ of the $\operatorname{arc} A$ which is a real 3-cell. Then $D-\operatorname{Int}(N)$ is a homotopy 3 -cell in $M$. Therefore, $D-\operatorname{Int}(N)$ is a real 3-cell. On the other hand, there is an isotopy of $D$ keeping all of it fixed except for a neighborhood of $N$ so that $D-\operatorname{Int}(N)$ image is $D$ itself; i.e., $D-\operatorname{Int}(N)$ is homeomorphic to $D$ itself. Therefore $D$ is a real cell.

\section{Main theorems.}

3.1. THEOREM. Let $p: E \rightarrow B$ be a Hurewicz fiber map from a connected separable metric ANR n-gm E over a principal ideal domain onto a wlc paracompact finite (covering) dimensional base space B. Suppose that all fibers are homeomorphic to a space $M$ where $M$ is a connected 3-manifold with exactly one end and whose one point compactification is a 3-manifold and all homotopy 3-cells in $M$ are 3-cells. Then the fibering $(E, B, p)$ is locally trivial. If $B$ is contractible then it is a product fiber space.

This theorem implies our original problem as a special case.

CoRollary 1. Let $p: E \rightarrow B$ be a Hurewicz fiber map from a connected separable metric ANR n-gm E over a principal ideal domain onto a wlc paracompact finite (covering) dimensional space $B$. If all fibers are homeomorphic to $R^{3}$, then the fibering $(E, B, p)$ is locally trivial. If $B$ is contractible, then it is a product fiber space.

Proof of Theorem 3.1. We note again that $B$ is necessarily 0 -connected, locally compact, separable metric, and ANR. Let $\bar{E}$ be the disjoint union of $E$ and the product space $B \times\{0\}$ and $\bar{p}: \bar{E} \rightarrow B$ be defined by $\bar{p}(e)=$ $p(e)$ for each $e \in E$ and $\bar{p}(e)=b$ for each $e=(b, 0) \in B \times\{0\}$. Let $\overline{\mathscr{U}}$ be the 
topology for $\bar{E}$ that is defined in $\S 2$. By Lemma $2.1, \bar{E}$ is a topologically complete metric space. Therefore there exist a complete metric space $E^{\prime}$ and a homeomorphism $h$ between $E^{\prime}$ and $\bar{E}$. If we define a map $p^{\prime}: E^{\prime} \rightarrow B$ to be $\bar{p} \cdot h$, then it is a homotopically 2-regular map from a complete metric space $E^{\prime}$ onto a finite (covering) dimensional metric space $B$ such that each inverse under $p^{\prime}$ is homeomorphic to a compact 3-manifold with boundary by Lemmas 2.1 and 2.2. Since each fiber $p^{-1}(b)$ has no homotopy 3-cells which are not real 3-cells, the space $\bar{p}^{-1}(b)$ that is the one point compactification of $p^{-1}(b)$ has no homotopy 3-cells which are not real 3-cells by Lemma 2.3. Therefore by (6.1) of [4], $p^{\prime}: E^{\prime} \rightarrow B$ is locally trivial. That is, for each $b \in B$, there exist an open set $U$ of $b$ in $B$ and a homeomorphism $h_{U}^{\prime}: U \times p^{\prime-1}(b) \rightarrow p^{\prime-1}(U)$ such that the diagram

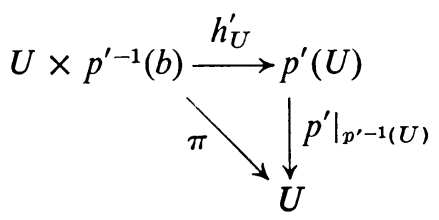

commutes, where $\pi$ is the projection map. Then the commutative diagram below gives us a trivialization of $\left.\bar{p}\right|_{U}: \bar{p}^{-1}(U) \rightarrow U$ :

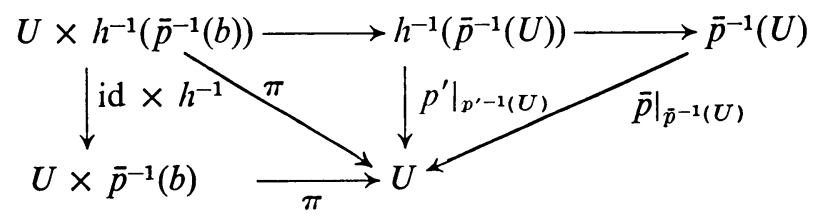

Therefore $\bar{p}: \bar{E} \rightarrow B$ is locally trivial. From this fact we can conclude that $p: E \rightarrow B$ itself is locally trivial. Since the proof of this is exactly the same as the one of the case where the dimension of the fiber is two [6, p. 63], we only give a sketch of the proof rather than a detailed proof.

Let

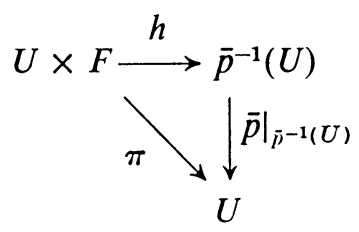

be a local trivialization of $\bar{p}: \bar{E} \rightarrow B$, where $F$ is the fiber. Suppose the image of $U \times\left(F-p^{-1}(b)\right)$ under $h$ is not equal to $\bar{p}^{-1}(U)-p^{-1}(U)$. (If it is equal, then nothing is to be done.) Define $f: U \rightarrow U \times F$ by $f(c)=\left(c, x_{0}\right), c \in U$, $x_{0}=F-p^{-1}(b)$, and $g: U \rightarrow \bar{p}^{-1}(U)$ by $g(c)=\bar{p}^{-1}(c)-p^{-1}(c), c \in U$. Then $h^{-1} g: U \rightarrow U \times F$ is different from $f$. We find an open set $V$ in $U$ and a fiber 
preserving homeomorphism $\phi: V \times F \rightarrow V \times F$ such that $\phi\left(h^{-1} g(V)\right)=f(V)$. Here we use the fact that the group of homeomorphisms of an $n$-disk $D^{n}$ fixing $\partial D^{n}$ and an interior point pointwise is locally contractible for all $n[\mathbf{1}]$. Then

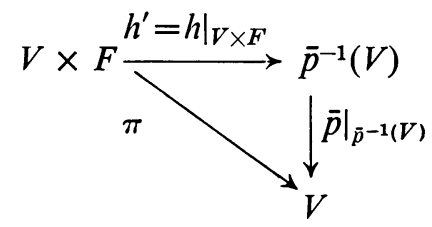

commutes. Therefore, $h^{\prime} \phi^{-1}:(V \times F, f(V)) \rightarrow\left(V \times F, h^{-1} g(V)\right) \rightarrow\left(\bar{p}^{-1}(V)\right.$, $g(V))=\left(\bar{p}^{-1}(V), \bar{p}^{-1}(V)-p^{-1}(V)\right)$ is a homeomorphism. Hence $h_{V}=$ $\left.h^{\prime} \phi^{-1}\right|_{V \times F-V \times\left(\bar{p}^{-1}(b)-p^{-1}(b)\right)}$ is a homeomorphism such that the diagram

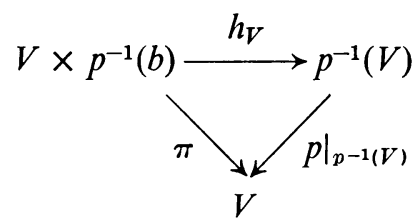

commutes. This is a local trivialization of the map $p: E \rightarrow B$. The last statement of the theorem is a result of the general bundle theory. Q.E.D.

We state an analogue theorem in Serre fibering.

3.2. THEOREM. Let $p: E \rightarrow B$ be a Serre fiber map from a connected separable metric ANR n-gm E over a principal ideal domain onto a wlc paracompact finite (covering) dimensional base space B. Suppose that all fibers are homeomorphic to a space $M$ where $M$ is a connected 3-manifold with exactly one end and whose one point compactification is a 3-manifold and all homotopy 3-cells in $M$ are 3-cells. Then the fibering $(E, B, p)$ is locally trivial. If $B$ is contractible then it is a product fiber space.

Proof. By Theorem 2 of [9], $p$ is homotopically $n$-regular; then by Ungar's theorem [13], $p$ is a Hurewicz fiber map Therefore the local triviality of the map follows by Theorem 3.1.

\section{REFERENCES}

1. J. W. Alexander, On the deformation of an h-cell, Proc. Nat. Acad. Sci. U.S.A. 9 (1923), 406-407.

2. E. Dyer and M.-E. Hamstrom, Completely regular mappings, Fund. Math. 45 (1958), 103-118. MR 19, 1187.

3. E. Fadell, On fiber spaces, Trans. Amer. Math. Soc. 90 (1959), 1-14. MR 21 \#330.

4. M.-E. Hamstrom, Regular mappings and the space of homeomorphisms on a 3manifold, Mem. Amer. Math. Soc. No. 40 (1961). MR 27 \#2970. 
5. M.-E. Hamstrom and E. Dyer, Regular mappings and the space of homeomorphisms on a 2-manifold, Duke Math. J. 25 (1958), 521-531. MR 20 \#2695.

6. S. K. Kim, Local triviality of Hurewicz fiber maps, Trans. Amer. Math. Soc. 135 (1969), 51-67. MR 38 \#1679.

7. - Local triviality of completely regular mappings, Duke Math. J. 38 (1971), 467-471.

8. S. L. Langston, Replacement and extension theorems in the theory of Hurewicz fiber spaces, Thesis, University of Wisconsin, Madison, Wis., 1968.

9. L. F. McAuley and P. A. Tulley, Fiber spaces and n-regularity, Topology Seminar (Wisconsin, 1965), Ann. of Math. Studies, no. 60, Princeton Univ. Press, Princeton, N.J., 1966, pp. 229-233. MR 35 \#7336.

10. F. Raymond, Local triviality for Hurewicz fiberings of manifolds, Topology 3 (1965), 43-57.

11. - The end point compactification of manifolds, Pacific J. Math. 10 (1960), 947-963. MR 22 \#11387.

12. S. B. Seidman, Completely regular mappings, Thesis, University of Michigan, Ann Arbor, Mich., 1969.

13. G. S. Ungar, Conditions for a mapping to have the slicing structure property, Pacific J. Math. 30 (1969), 549-553. MR 40 \#3550.

14. R. L. Wilder, Topology of manifolds, Amer. Math. Soc. Colloq. Publ., vol. 32, Amer. Math. Soc., Providence, R.I., 1949. MR 10, 614.

Department of Mathematics, University of Connecticut, Storrs, Connecticut 06268 